\title{
Respiratory Sinus Arrhythmia as an Index of Cardiac Vagal Control in Mitral Valve Prolapse
}

\section{Lucia BONA OLEXOVA ${ }^{1,2}$, Nikola SEKANINOVA ${ }^{1,2}$, Alexander JURKO JR ${ }^{3}$, Zuzana VISNOVCOVA $^{2,1}$, Marian GRENDAR ${ }^{2}$, Tomas JURKO ${ }^{4}$, Ingrid TONHAJZEROVA ${ }^{1,2}$}

${ }^{1}$ Department of Physiology, Jessenius Faculty of Medicine in Martin, Comenius University Bratislava, Martin, Slovak Republic, ${ }^{2}$ Biomedical Center Martin, Jessenius Faculty of Medicine in Martin, Comenius University Bratislava, Martin, Slovak Republic, ${ }^{3}$ Paediatric Cardiology, Jessenius Faculty of Medicine in Martin, Comenius University Bratislava, Martin, Slovak Republic, ${ }^{4}$ Neonatology Clinic, Jessenius Faculty of Medicine in Martin, Comenius University Bratislava, University Hospital Martin, Martin, Slovak Republic

Received August 27, 2019

Accepted November 8, 2019

\begin{abstract}
Summary
Respiratory sinus arrhythmia (RSA), i.e. heart rate (HR) variations during inspiration and expiration, is considered as a noninvasive index of cardiac vagal control. Mitral valve prolapse (MVP) could be associated with increased cardiovascular risk; however, the studies are rare particularly at adolescent age. Therefore, we aimed to study cardiac vagal control indexed by RSA in adolescent patients suffering from MVP using short-term heart rate variability (HRV) analysis. We examined 12 adolescents (girls) with MVP (age $15.9 \pm 0.5$ years) and 12 age and gender matched controls. Resting ECG was continuously recorded during 5 minutes. Evaluated HRV indices were RR interval (ms), rMSSD (ms), pNN50 (\%), log HF $\left(\mathrm{ms}^{2}\right)$, peak $\mathrm{HF}(\mathrm{Hz})$ and respiratory rate (breaths/min). RR interval was significantly shortened in MVP group compared to controls $(p=0.004)$. HRV parameters-rMSSD, pNN50 and log HF were significantly lower in MVP compared to controls $(p=0.017$, $p=0.014, p=0.015$ respectively). Our study revealed reduced RSA magnitude indicating impaired cardiac vagal control in MVP already at adolescent age that could be crucial for early diagnosis of cardiovascular risk in MVP.
\end{abstract}

\section{Key words}

Respiratory sinus arrhythmia • Cardiac vagal control • Mitral valve prolapse • Adolescents

\section{Corresponding author}

Ingrid Tonhajzerova, Department of Physiology and Biomedical Center Martin, Jessenius Faculty of Medicine in Martin, Comenius
University in Bratislava, Mala Hora 4C, 03601 Martin, Slovak Republic. E-mail: ingrid.tonhajzerova@jfmed.uniba.sk

\section{Introduction}

Breathing and cardiac activity are tightly coupled resulting in heart rate variations according to respiratory pattern-heart rate (HR) increases during the inspiratory phase and vice versa, HR declines during the expiratory phase. This physiological phenomenon known as respiratory sinus arrhythmia (RSA) is the result of multiple central and peripheral mechanisms. From physiological view, vagally-mediated cardioinhibitory center represented by nc. ambiguus is influenced by breathing via many pathways involving the interaction between cardioinhibitory and respiratory centers (Chen et al. 2017), activation of the pulmonary stretch receptors associated with lung inflation, or reflex responses to the changes in intrathoracic and blood pressure changes (Mortola et al. 2016, Langer et al. 2018). However, cardiac-linked vagal efferent modulation from the $n c$. ambiguus is influenced by several brain regions included also in cognitive and emotional processing (e.g. amygdala, cingulate, prefrontal cortex), therefore, the RSA is considered as a noninvasive marker of cognitive/emotional regulation in psychophysiological research. Taken together, this complex cardio-respiratory regulatory network modulates both preganglionic vagal and sympathetic motoneurons 
resulting in respiratory-linked "beat-to-beat" $\mathrm{HR}$ oscillations-short-term heart rate variability (HRV) determined mainly by vagal efferent outflow (Thayer and Lane 2000, 2009). Moreover, objective neuroimaging studies revealed that respiratory-linked short-term HRV is associated with several central regions such as prefrontal or cingulate cortex (Lane et al. 2009, Thayer et al. 2012).

RSA can be quantified by short-term HRV linear analysis providing information about respiration-related effects on the high-frequency band (HF-HRV). Recent study noted that RSA quantified by various HRV timedomain and frequency-domain analysis could provide important information about discrete abnormalities in cardiac vagal control (Mestanik et al. 2019).

Mitral valve prolapse (MVP) characterized by one or both abnormal mitral leaflets in the left atrium contributing to mitral regurgitation represents common defect afflicting 2-3\% of world population that is manifested by late systolic murmur (Hu and Zhao 2011, Delling and Vasan 2014). The most common symptoms coupling with MVP are palpitations, orthostatic rhythm disorder, exertional dyspnoea, anomalous chest pain, and neuropsychiatric symptoms (e.g. anxiety) (Delling and Vasan 2014).

With respect to MVP-linked autonomic regulation, current studies focus mainly on sympathetic branch revealing elevated concentrations of catecholamines in circulation and enhanced $\beta$ receptor affinity indicating the presence of hyperadrenergic state (Hu and Zhao 2011, Hu et al. 2014). Additionally, several studies emphasized the role of respiratory-linked cardiac vagal regulation (indexed by short-term HRV) in assessment of cardiovascular risk (Jíra et al. 2010). In this aspect, autonomic sympathovagal imbalance - decreased parasympathetic activity associated with increased sympathetic activity - can lead to various diseases associated with higher cardiovascular risk also in MVP (e.g. hypertension, tachycardia, procoagulation, metabolic syndrome) (Hu and Zhao 2011, Abboud et al. 2012, Licht et al. 2013, Garafova et al. 2014, Mestanik et al. 2015). Despite the fact that MVP is characterized by sympathetic overactivity, the cardio-respiratory coupling indexed by RSA could be crucial in the early diagnosis of cardiovascular risk in MVP already at vulnerable adolescent age. However, this question is still unclear.

We addressed the hypothesis that MVP could be associated with impaired HR autonomic regulation already in adolescents, therefore, we aimed to study cardiac vagal control indexed by RSA in adolescents suffering from
MVP. To the best of our knowledge, this is the first study to assess cardiac chronotropic regulation using short-term HRV analysis in MVP at adolescent age.

\section{Methods}

The study was approved by the Ethics Committee of Jessenius Faculty of Medicine in Martin, Comenius University in Bratislava in accordance with the 1964 Helsinki declaration and its later amendments. All subjects and their parents were carefully instructed about the study protocol and they gave informed written consent to participation in the study prior to the examination.

\section{Subjects}

We examined 12 girls with MVP (age: $15.9 \pm 0.5$ years, body mass index: $18.2 \pm 0.6 \mathrm{~kg} / \mathrm{m}^{2}$ ) and age $/$ gendermatched healthy girls as control group (age: $15.9 \pm 0.5$ years, body mass index: $20.5 \pm 0.6 \mathrm{~kg} / \mathrm{m}^{2}$ ). The subjects suffering from MVP were recruited from specialized Pediatric cardiology, external workplace of Jessenius Faculty of Medicine in Martin. The MVP was diagnosed by specialist in pediatric cardiology.

MVP was suspected according to the presence of typical midsystolic click during auscultation, which occurred alone or coupled with telesystolic murmur. All patients were characterized by symptoms of palpitations, syncope, chest pain or fatigue. Additionally, the nonspecific changes, e.g. flat or inverted T waves in II, III and aVF leads, were present on ECG recording. The final MVP diagnosis was confirmed by cross-sectional echocardiography, showing prolapse of one or both mitral leaflets in the long-axis view and four-chamber view. Moreover, Doppler echocardiography revealed mitral regurgitation in all patients with MVP.

The exclusion criteria for both MVP and control group were following: smoking, overweight and obesity, history of recent acute illness or respiratory, endocrinological, neurological, metabolic, or infectious diseases or mental disorders. Only participants without pharmacological treatment were enrolled in the study.

\section{Study protocol}

Examinations were performed in a quiet room under standard conditions (temperature: $22-23{ }^{\circ} \mathrm{C}$, humidity: $45-55 \%$ ), with the minimization of stimuli, in the morning after normal breakfast, between 8:00 and 12:00 a.m. Before the examination, the participants were instructed to avoid physical exercise at least $24 \mathrm{~h}$ prior to the examination. 
The subjects were asked to sit comfortably in a special armchair. Before examination participants rest $15 \mathrm{~min}$, ensuring autonomic nervous system stabilization and for the exclusion of potential effect of stress. Afterwards, participants remained in sitting position and continuous $5 \mathrm{~min}$ recordings of the time series of RRintervals were performed using Polar V800 (Polar Electro, Kempele, Finland) with sampling frequency $1000 \mathrm{~Hz}$.

\section{HRV data analyses}

The time series of RR-intervals were derived from the continuous ECG-recordings and carefully checked for the presence of artefacts. The artefact-free 5 min sequences of RR-intervals were analyzed by means of time- and frequency-domain methods using HRV analysis software package HRVAS (Ramshur 2010).

\section{Time-domain analysis}

In a time-domain analysis based on the calculation of beat-to-beat differences in the duration of RR-intervals, root mean square of successive differences (rMSSD, ms) and percentage of the number of successive heartbeats differing more than $50 \mathrm{~ms}$ (pNN50, \%) were calculated. Parameters rMSSD and pNN50 are considered to represent the time-domain indices of RSA magnitude (Task Force 1996).

\section{Frequency-domain analysis}

Prior to HRV frequency-domain analysis, the slow fluctuations were filtered using smoothness priors detrending with $\Lambda=500$ (Tarvainen et al. 2002). Consequently, the spectral power in high-frequency band of HRV (HF-HRV, 0.15-0.40 Hz) was analyzed using frequency-domain parametric method of autoregressive (AR) spectral estimation using Burg periodogram. For the frequency-domain analyses using Burg periodograms, the time series of RR intervals were resampled using cubic spline interpolation with the frequency $4 \mathrm{~Hz}$ to meet the assumption of a regularly time-sampled signal. Estimation of HF-HRV with Burg periodogram (AR) was performed using model order 16, which is recommended for the short series of RR intervals with the sampling frequency $2-4 \mathrm{~Hz}$ (Boardman et al. 2002). Peak frequency within the highfrequency band (peak HF, Hz) was assessed by AR method HF-HRV analysis and the respiratory rate (breaths, $\mathrm{min}^{-1}$ ) was calculated on the basis of respiratory-related $\mathrm{HF}$ frequency (Visnovcova et al. 2014).

\section{Statistical analysis}

Data were analyzed using statistical software package Systat (Systat Software Inc., USA) and R (R Foundation for Statistical Computing, Vienna, Austria 2015), version 3.5.2. Data and basic descriptive statistics (median, lower and upper quartile) were visualized by boxplot, overlaid with swarmplot. Because the HRV parameter HF-HRV had positively skewed distribution, their values were logarithmically transformed for statistical testing. Normality assumption was assessed by the quantile-quantile plot with the $95 \%$ confidence band, constructed by bootstrap. When the assumption of normality was not tenable, the Wilcoxon two sample test was used to test the hypothesis of the equality of the population medians of the quantity in question, in cases and controls. Otherwise, the t test was used to test the hypothesis of the population means. Results of test with the p-value below 0.05 are considered statistically significant. All data are expressed as mean $\pm \mathrm{SE}$.

\section{Results}

Statistical analysis revealed significantly shortened RR interval in MVP group compared to controls (638.11 ms vs $788.44 \mathrm{~ms}, \mathrm{p}=0.004)$. The RSA magnitude indexed by HRV time- and frequency-domain parametersrMSSD, pNN50 and log HF - was significantly reduced in MVP group compared to controls ( $\mathrm{p}=0.017, \mathrm{p}=0.014$, $\mathrm{p}=0.015$ respectively). No significant differences were found in the remaining HRV parameters (peak HF, respiratory rate).

Body mass index (BMI) was significantly lower in MVP group compared to controls $\left(18.22 \mathrm{~kg} / \mathrm{m}^{2}\right.$ vs 20.51 $\left.\mathrm{kg} / \mathrm{m}^{2}, \mathrm{p}=0.028\right)$. The results are summarized in the Table 1 .

\section{Discussion}

Our study revealed reduced magnitude of RSA indexed by HRV short-term analysis in adolescents with MVP compared to controls indicating impaired cardiac vagal regulation. It seems that discrete abnormal cardiorespiratory coupling indexed by RSA is associated with higher cardiovascular risk in MVP already at adolescent age. These findings are in accordance with study by Lin Han et al. (2000), who discovered reduced RSA magnitude indexed by HRV long-term analysis in children suffering from MVP. However, 24-hour Holter recordings used in 
Table 1. HRV parameters

\begin{tabular}{|c|c|c|c|}
\hline Parameters & $\begin{array}{c}\text { Control } \\
(n=12)\end{array}$ & $\begin{array}{c}\text { MVP } \\
(n=12)\end{array}$ & p-value \\
\hline Age (years) & $15.92 \pm 0.51$ & $15.92 \pm 0.51$ & nonsignificant \\
\hline$B M I\left(k g / m^{2}\right)$ & $20.51 \pm 0.69$ & $18.22 \pm 0.62$ & 0.028 \\
\hline \multicolumn{4}{|l|}{ HRV parameters } \\
\hline \multicolumn{4}{|l|}{ Time domain analysis: } \\
\hline RR-interval (ms) & $788.44 \pm 39.53$ & $638.11 \pm 19.65$ & 0.004 \\
\hline$r M S S D(m s)$ & $51.26 \pm 9.08$ & $23.38 \pm 3.33$ & 0.017 \\
\hline$p N N 50(\%)$ & $25.40 \pm 6.88$ & $5.63 \pm 2.51$ & 0.014 \\
\hline \multicolumn{4}{|l|}{ Frequency domain analysis: } \\
\hline $\log H F H R V\left(m s^{2}\right)$ & $6.66 \pm 0.36$ & $5.46 \pm 0.27$ & 0.015 \\
\hline peak $H F(H z)$ & $0.23 \pm 0.02$ & $0.28 \pm 0.02$ & 0.065 \\
\hline Respiratory rate (/min) & $13.75 \pm 0.99$ & $16.85 \pm 1.24$ & 0.073 \\
\hline
\end{tabular}

BMI-body mass index, rMSSD-root mean square of successive differences of RR-intervals, pNN50-percentage of the number of successive RR-intervals differing more than $50 \mathrm{~ms}$, log HF-logarithmic value of spectral power in high frequency band of heart rate, peak HF-peak frequency within high-frequency band of heart rate variability. Values are expressed as mean $\pm \mathrm{SE}$.

this study for HRV analysis are influenced by numerous factors, e.g. diurnal rhythm-linked autonomic regulatory changes, hormonal changes, or daily activities (Kim et al. 2018). Thus, the information related to alone cardiac autonomic regulation-specifically about cardio-respiratory coupling - is limited. In contrast, resting short-term HRV is determined mainly by vagal efferent outflow, thus, the respiratory-linked high frequency band of $\mathrm{HRV}$ is considered as a noninvasive marker of cardiac vagal control (Berntson et al. 1997, Thayer and Lane 2000, 2009, Laborde et al. 2017). However, the pathway linking abnormal cardiovagal regulation and MVP found in our study is still unclear. Several mechanisms are suggested.

Firstly, hemodynamic changes related to MVP could represent an important factor influencing chronotropic regulation of heart activity. Specifically, the MVP can be associated with mitral regurgitation, or the MVP is diagnosed without mitral regurgitation. In our study, all patients suffering from MVP were characterized by mitral regurgitation, therefore, mitral regurgitationlinked hemodynamic changes (increased pulmonary capillary wedge pressure and volume overload) (Bakkestrøm et al. 2018) may lead to increased volume and pressure overload in right atrium affecting sinoatrial (SA) node as the primary pacemaker (Cooper and Kohl 2005). In this context, local heamodynamically-mediated SA distension could represent one of the pathomechanisms leading to impaired heart rate control in MVP.

Further, HR control is under cortical tonic inhibitory control peripherally via the vagus (Levy 1990, Uijtdehaage and Thayer 2000). From this perspective, afferent-efferent vagal pathways involved in HR control are regulated via central autonomic network (CAN). Structurally, CAN represents integrated component consisting of several interconnected cortical as well as subcortical brain areas through which the brain controls visceromotor, neuroendocrine and behavioral responses (the anterior cingulate, insular, and ventromedial prefrontal cortices, the central nucleus of the amygdala, the paraventricular and related nuclei of the hypothalamus, the periaqueductal gray matter, the parabrachial nucleus, the nucleus of the solitarii tract, the nucleus ambiguus, the ventromedial and ventrolateral medulla, the medullary tegmental field) (Benarroch 1993). According to the neurovisceral integration model, the CAN structures are overlapping with brain areas involved also in attentional, cognitive and emotional network (Thayer and Lane 2000). Taken together, RSA magnitude indexed by short-term HRV could be considered as an index of cardiac vagal control as well as cognitive/emotional regulation (Thayer and Lane 2000, Grossman et al. 2004).

Importantly, the effective functioning of prefrontal-subcortical inhibitory circuits is crucial for emotional and self-regulation (Thayer and Lane 2000, 
Thayer et al. 2009). Moreover, several pharmacological and neuroimaging studies have shown a positive association between the activation of inhibitory prefrontalsubcortical circuits and cardiac vagal control as reflected by resting HRV (Ahern et al. 2001, Lane et al. 2009). In other words, under physiological circumstances the prefrontal cortex exerts its inhibitory control over sympathoexcitatory subcortical circuits resulting in high cardiac vagal control. In contrast, impaired prefrontal activity is associated with disruption of inhibitory functioning resulting in dominance of sympathoexcitatory subcortical circuits and reduced cardiovagal modulation (Park et al. 2013, Thayer et al. 2009, Park and Thayer 2014). With respect to MVP, recent studies found emotional and cognitive dysregulation in patients with mitral regurgitation (Zinchenko and Pervichko 2014, Sung et al. 2019). It seems that disruption of prefrontal cortex inhibitory functioning linked to emotional/cognitive dysregulation could represent an important pathomechanism leading to diminished cardiovagal control in MVP. Thus, it is questionable whether our findings of lower cardiac vagal modulation are predominantly related to the MVP hemodynamicallylinked features or it is the reflection of discrete impairment of complex neurocardiac integrity in MVP already at adolescent age.

\section{Conclusion}

Our study revealed MVP-linked reduced RSA magnitude indicating abnormal cardiac vagal control that could be associated with higher cardiovascular risk in MVP already at adolescent age. We suggest that evaluation of RSA magnitude using short-term HRV analysis could be helpful for early diagnosis of cardiovascular risk in MVP.

\section{Limitations}

The studied group consisting only of female patients with MVP is relatively small. In this context, age and gender could represent an important factor influencing autonomic nervous system activity (Chang et al. 2016), therefore, our findings cannot be extrapolated to general population and need to be independently validated in larger groups of patients with respect to gender differences.

\section{Conflict of Interest}

There is no conflict of interest.

\section{Acknowledgements}

This work was supported by the Slovak Scientific Grant Agency (VEGA 1/0044/18, VEGA 1/0190/20) and the project "Biomedical Center Martin" (ITMS 26220220187) co-financed from EU sources.

\section{References}

ABBOUD FM, HARWANI SC, CHAPLEAU MW: Autonomic neural regulation of the immune system: implications for hypertension and cardiovascular disease. Hypertension 59: 755-762, 2012. https://doi.org/10.1161/hypertensionaha.111.186833

AHERN GL, SOLLERS JJ, LANE RD, LABINER DM, HERRING AM, WEINAND ME, HUTZLER R, THAYER JF: Heart rate and heart rate variability changes in the intracarotid sodium amobarbital test. Epilepsia 42: 912-921, 2001. https://doi.org/10.1046/j.1528-1157.2001.042007912.X

BAKKESTRØM R, BANKE A, CHRISTENSEN NL, PECINI R, IRMUKHAMEDOV A, ANDERSEN M, BORLAUG BA, MØLLER JE: Hemodynamic characteristics in significant symptomatic and asymptomatic primary mitral valve regurgitation at rest and during exercise. Circ Cardiovasc Imaging 11: e007171, 2018. https://doi.org/10.1161/circimaging.117.007171

BENARROCH EE: The central autonomic network: functional organization, dysfunction, and perspective. Mayo Clin Proc 68: 988-1001, 1993. https://doi.org/10.1016/s0025-6196(12)62272-1

BERNTSON GG, BIGGER JT, ECKBERG DL, GROSSMAN P, KAUFMANN PG, MALIK M, NAGARAJA HN, PORGES SW, SAUL JP, STONE PH, VAN DER MOLEN MW: Heart rate variability: Origins, methods, and interpretive caveats. Psychophysiology 34: 623-648, 1997. https://doi.org/10.1111/j.1469-8986.1997.tb02140.x

BOARDMAN A, SCHLINDWEIN FS, ROCHA AP, LEITE A: A study on the optimum order of autoregressive models for heart rate variability. Physiol Meas 23: 325-336, 2002. https://doi.org/10.1088/0967-3334/23/2/308 
CHEN Z, CHEN H, CHEN F, GU D, SUN L, ZHANG W, FAN L, LIN Y, DONG R, LAI K: Vagotomy decreases the neuronal activities of medulla oblongata and alleviates neurogenic inflammation of airways induced by repeated intra-esophageal instillation of $\mathrm{HCl}$ in guinea pigs. Physiol Res 66: 1021-1028, 2017. https://doi.org/10.33549/physiolres.933574

COOPER PJ, KOHL P: Species- and preparation-dependence of stretch effects on sino-atrial node pacemaking. Ann NY Acad Sci 1047: 324-335, 2005. https://doi.org/10.1196/annals.1341.029

DELLING FN, VASAN RS: Epidemiology and pathophysiology of mitral valve prolapse: new insights into disease progression, genetics, and molecular basis. Circulation 129: 2158-2170, 2014. https://doi.org/10.1161/circulationaha.113.006702

GARAFOVA A, PENESOVA A, CIZMAROVA E, MARKO A, VLCEK M, JEZOVA D: Cardiovascular and sympathetic responses to a mental stress task in young patients with hypertension and/or obesity. Physiol Res 63 (Suppl 4): S459-S467, 2014.

GROSSMAN P, WILHELM FH, SPOERLE M: Respiratory sinus arrhythmia, cardiac vagal control, and daily activity. Am J Physiol Heart Circ Physiol 287: H728-H734, 2004.

HAN L, HO TF, YIP WC, CHAN KY: Heart rate variability of children with mitral valve prolapse. J Electrocardiol 33 : 219-224, 2000. https://doi.org/10.1054/jelc.2000.7661

HU X, WANG HZ, LIU J, CHEN AQ, YE XF, ZHAO Q: A novel role of sympathetic activity in regulating mitral valve prolapse. Circ J 78: 1486-1493, 2014. https://doi.org/10.1253/circj.cj-13-1222

HU X, ZHAO Q: Autonomic dysregulation as a novel underlying cause of mitral valve prolapse: a hypothesis. Med Sci Monit 17: 27-31, 2011. https://doi.org/10.12659/msm.881918

JÍRA M, ZÁVODNÁ E, NOVÁKOVÁ Z, FISER B, HONZÍKOVÁ N: Reproducibility of blood pressure and inter-beat interval variability in man. Physiol Res 59: 113-121, 2010.

KIM HG, CHEON EJ, BAI DS, LEE YH, KOO BH: Stress and heart rate variability: a meta-analysis and review of the literature. Psychiatry Investig 15: 235-245, 2018. https://doi.org/10.30773/pi.2017.08.17

LABORDE S, MOSLEY E, THAYER JF: Heart rate variability and cardiac vagal tone in psychophysiological research - recommendations for experiment planning, data analysis, and data reporting. Front Psychol 8: $213,2017$. https://doi.org/10.3389/fpsyg.2017.00213

LANE R, MCRAE K, REIMAN E, CHEN K, AHERN G, THAYER J: Neural correlates of heart rate variability during emotion. Neuroimage 44: 213-222, 2009. https://doi.org/10.1016/j.neuroimage.2008.07.056

LANGER P, JURÁK P, VONDRA V, HALÁMEK J, MEŠŤANÍK M, TONHAJZEROVÁ I, VIŠČOR I, SOUKUP L, MATEJKOVA M, ZÁVODNÁ E, LEINVEBER P: Respiratory-induced hemodynamic changes measured by whole-body multichannel impedance plethysmography. Physiol Res 67: 571-581, 2018. https://doi.org/10.33549/physiolres.933778

LEVY MN: Autonomic interactions in cardiac control. Ann N Y Acad Sci 601: 209-221, 1990.

LICHT CMM, DE GEUS EJC, PENNINX BWJH: Dysregulation of the autonomic nervous system predicts the development of the metabolic syndrome. J Clin Endocrinol Metab 98: 2484-2493, 2013. https://doi.org/10.1210/jc.2012-3104

MESTANIK M, MESTANIKOVA A, LANGER P, GRENDAR M, JURKO A, SEKANINOVA N, VISNOVCOVA N, TONHAJZEROVA I: Respiratory sinus arrhythmia - testing the method of choice for evaluation of cardiovagal regulation. Respir Physiol Neurobiol 259: 86-92, 2019. https://doi.org/10.1016/j.resp.2018.08.002

MESTANIK M, MESTANIKOVA A, VISNOVCOVA Z, CALKOVSKA A, TONHAJZEROVA I: Cardiovascular sympathetic arousal in response to different mental stressors. Physiol Res 64: 585-594, 2015.

MORTOLA JP, MARGHESCU D, SIEGRIST-JOHNSTONE R: Thinking about breathing: Effects on respiratory sinus arrhythmia. Respir Physiol Neurobiol 223: 28-36, 2016. https://doi.org/10.1016/j.resp.2015.12.004

PARK G, THAYER JF: From the heart to the mind: cardiac vagal tone modulates top-down and bottom-up visual perception and attention to emotional stimuli. Front Psychol 5: 278, 2014. https://doi.org/10.3389/fpsyg.2014.00278

PARK G, VASEY MW, VAN BAVEL JJ, THAYER JF: Cardiac vagal tone is correlated with selective attention to neutral distractors under load. Psychophysiology 50: 398-406, 2013. https://doi.org/10.1111/psyp.12029 
RAMSHUR JT: Design, Evaluation, and application of heart rate variability analysis software (HRVAS). The University of Memphis. 2010.

SUNG SH, LEE CW, WANG PN, LEE HY, CHEN CH, CHUNG CP: Cognitive functions and jugular venous reflux in severe mitral regurgitation: A pilot study. PLoS One 14: e0207832, 2019. https://doi.org/10.1371/journal.pone.0207832

TARVAINEN MP, RANTA-AHO PO, KARJALAINEN PA: An advanced detrending method with application to HRV analysis. IEEE Trans Biomed Eng 49: 172-175, 2002. https://doi.org/10.1109/10.979357

TASK FORCE OF THE EUROPEAN SOCIETY OF CARDIOLOGY AND THE NORTH AMERICAN SOCIETY OF PACING AND ELECTROPHYSIOLOGY: Heart rate variability: standards of measurement, physiological interpretation and clinical use. Circulation 93: 1043-1065, 1996. https://doi.org/10.1161/01.cir.93.5.1043

THAYER JF, ÅHS F, FREDRIKSON M, SOLLERS JJ, WAGER TD: A meta-analysis of heart rate variability and neuroimaging studies: Implications for heart rate variability as a marker of stress and health. Neurosci Biobehav Rev 36: 747-756, 2012. https://doi.org/10.1016/j.neubiorev.2011.11.009

THAYER JF, HANSEN AL, SAUS-ROSE E, JOHNSEN BH: Heart rate variability, prefrontal neural function, and cognitive performance: the neurovisceral integration perspective on self-regulation, adaptation, and health. Ann Behav Med 37: 141-153, 2009. https://doi.org/10.1007/s12160-009-9101-z

THAYER JF, LANE RD: A model of neurovisceral integration in emotion regulation and dysregulation. J Affect Disord 61: 201-216, 2000. https://doi.org/10.1016/s0165-0327(00)00338-4

THAYER JF, LANE RD: Claude Bernard and the heart-brain connection: Further elaboration of a model of neurovisceral integration. Neurosci Biobehav Rev 33: 81-88, 2009. https://doi.org/10.1016/j.neubiorev.2008.08.004

UIJTDEHAAGE SH, THAYER JF: Accentuated antagonism in the control of human heart rate. Clin Auton Res 10: 107110, 2000. https://doi.org/10.1007/bf02278013

VISNOVCOVA Z, MESTANIK M, JAVORKA M, MOKRA D, GALA M, JURKO A, CALKOVSKA A, TONHAJZEROVA I: Complexity and time asymmetry of heart rate variability are altered in acute mental stress. Physiol Meas 35: 1319-1334, 2014. https://doi.org/10.1088/0967-3334/35/7/1319

ZINCHENKO YP, PERVICHKO EI: Qualitative characteristics of emotion regulation process in adolescents with mitral valve prolapse. Procedia - Soc Behav Sci 146: 76-82, 2014. https://doi.org/10.1016/j.sbspro.2014.08.089 